\title{
ХИРУРГИЧЕСКАЯ ТАКТИКА ПРИ ПЕРФОРАТИВНЫХ ЯЗВАХ ЖЕЛУДКА И ДВЕНАДЦАТИПЕРСТНОЙ КИШКИ
}

\section{SURGICAL TACTICS FOR PERFORATIVE ULCERS STOMACH AND DUODENUM}

\author{
M. Omarov \\ M. Magomedov \\ M. Magomedov
}

Summary. Target. Evaluation of the effectiveness of surgical treatment of perforated gastric and duodenal ulcers in a comparative aspect using various methods.

Materials and methods. Clinical studies of the results of treatment of 117 patients with perforated gastric and duodenal ulcers were carried out in a comparative aspect using laparoscopic and open methods of surgical treatment. Among the operated were 98 (81.7\%) men and 22 (18.3\%) women, aged from 21 to 65 years. Indications for laparoscopic suturing were established in 56 (46.7\%) patients and for open surgery - in 61 $(50.8 \%)$ patients. The study included a patient with a perforated ulcer within $2 \mathrm{~cm}$. Outcome indicators were determined according to length of stay, postoperative complications and mortality.

Results. All 117 patients met the pre-established study inclusion criteria. The mean surgery time was slightly longer in the laparoscopy group compared to the open surgery group (74.01 and 56.17 minutes, respectively). The average need for postoperative analgesia, mobilization time and hospital stay were significantly shorter after laparoscopy than after open surgery (1.21 days, 9.32 hours and 3.12 days; versus 3.83 days, 16.20 hours and 4,85 days, respectively). Postoperative complications were observed in $6(10.7 \%)$ patients in the laparoscopy group and in 24 (39.3\%) patients in the open surgery group.

Conclusions. Laparoscopic ulcer suturing is a safe approach and more effective than open surgery in terms of the need for postoperative analgesia, mobilization, length of hospital stay, and incidence of postoperative respiratory and wound complications.

Keywords: laparoscopy, perforation, perforated duodenal ulcer, suturing.

\author{
Омаров Магомед Дибирович \\ Аспирант, Дагестанский государственный \\ медицинский университет \\ maga05region1994@mail.ru \\ Магомедов Мухума Магомедович \\ Д.м.н., профессор, Дагестанский государственный \\ медицинский университет \\ muxuma@mail.ru \\ Магомедов Магомед Абдулхаликович \\ Д.м.н., доцент, Дагестанский государственный \\ медицинский университет \\ Drmda08@mail.ru
}

Аннотация. Цель. Оценка эффективности оперативного лечения перфоративных язв желудка и двенадцатиперстной кишки в сравнительном аспекте с применением различных методов.

Материалы и методы. Проведены клинические исследования результатов лечения 117 пациентов с перфоративной язвой желудка и двенадцатиперстной кишки в сравнительном аспекте с применением лапароскопических и открытых методов оперативного лечения. (реди оперированных было $98(81,7 \%)$ мужчин и 22 (18,3\%) женщин, возраст от 21 до 65 лет. Показания к лапароскопическому ушиванию были установленны у $56(46,7 \%)$ и к открытому оперативному вмешательству - у 61 (50,8\%) пациентов. В исследование включены пациента с перфоративной язвой размером в пределах 2 см. Показатели исхода определялись в соответствии с продолжительностью пребывания, послеоперационными осложнениями и летальностью.

Результаты. Все 117 пациентов соответствовали заранее установленным критериям включения исследования. Среднее время операции было несколько больше в группе лапароскопии по сравнению с группой открытой операции (74,01 и 56,17 минуты, соответственно). (редняя потребность в послеоперационной анальгезии, время мобилизации и пребывание в стационаре были значительно короче после лапароскопии, чем после открытой операции (1,21 дня, 9,32 часа и 3,12 дня; против 3,83 дня, 16,20 часа и 4,85 дня, соответственно). У 6 (10,7\%) пациентов в группе лапароскопии и у 24 (39,3\%) в группе открытой операции наблюдались послеоперационные осложнения.

Выводы. Лапароскопическое ушивание язвы является безопасным подходом и более эффективным, чем открытая операция, с точки зрения необходимости послеоперационной анальгезии, мобилизации, продолжительности пребывания в стационаре и частоты послеоперационных респираторных и раневых осложнений.

Ключевые слова: лапароскопия, перфорация, перфоративная язва двенадцатиперстной кишки, ушивание. 


\section{Ввемение}

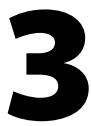
а последние десятилетия произошли значительные изменения в подходе к абдоминальным операциям с внедрением и усовершенствованием минимально инвазивных методик оперативных вмешательств. Лапароскопия в настоящее время стала золотым стандартом для большинства абдоминальных вмешательств [1,2]. Тем не менее, до сих пор продолжается дискуссия о целесообразности использования лапароскопического подхода в чрезвычайных ситуациях, таких как перитонит. Ранее опубликованные исследования предупреждают о пневмоперитонеум-индуцированной бактериемии и замедленном выздоровлении $[3,4]$.

Перфорация гастродуодиальных язв (ПГДЯ) является тяжелым осложнением и связана с госпитальной летальностью 5-25\% [1-4]. Методика лапароскопического ушивания при перфорации гастродуоденальных язв была введена два десятилетия назад. Самые ранние проспективные исследования продемонстрировали безопасность и целесообразность лапароскопической пластики $[5,4]$. Хотя ранние результаты были впечатляющими, исследования имели ряд ограничений: предвзятость отбора (сообщалось только о пациентах с неосложненными язвами, но с высокими показателями конверсии (14-23\%), предвзятость производительности (опыт хирургов не был четко определен) и низкую статистическую мощность.

Исследования показали, что сообщения о лапароскопической технологии (ЛТ) включали пациентов с ранним предлежанием и небольшим размером перфорации, и остается неясным, может ли ЛТ применяться ко всем пациентам [6]. Также ЛТ может быть технически сложной задачей без определенного уровня обучения и навыков. Было показано, что лапаротомия вызывает значительную физиологическую стрессовую реакцию, которая может привести к значительным осложнениям [6], а экстренная лапаротомия связана с 5\% смертностью, 20\% осложнений и $3 \%$ долгосрочным риском непроходимости кишечника [7].

Мы считаем, что политика селективного применения ЛТ позволит снизить число осложнений и улучшить периоперационные исходы. Ранние опыты показали, что Лт может быть успешно выполнена при наличии у пациентов перфорации размером <10 мм, расположенной в пилородуоденальной области, с оценкой по шкале Воеу 0-1 балла и гемодинамически стабильной [8].

В настоящее время нет никаких доказательств того, что ЛТ является более совершенным подходом по срав- нению с открытым методом. В недавнем метаанализе установлено, что лапароскопическая технология несколько выгодна с точки зрения уменьшения послеоперационной боли и более короткой продолжительности пребывания пациентов в стационаре [9]. Однако авторы посчитали, что исследования, включенные в этот анализ, несмотря на то, что они были недавними и качественными, не имеют однородного сравнения, особенно среди неслучайных исследований, обнаружено, что они смещены в сторону отбора более молодых пациентов мужского пола в группе ЛТ. Поэтому мы считаем, что существует необходимость в сравнении сопоставимых когорт для точной оценки хирургического подхода и исходов. Настоящее исследование представляет собой попытку сравнить краткосрочные исходы между лапароскопической технологией (Лт) и открытым методом (ОМ). Вторичная цель состоит в том, чтобы проанализировать послеоперационные осложнения и смертность между ЛТ и ОМ в зависимости от тяжести перитонита.

\section{Шель исслеАования}

Оценка эффективности оперативного лечения перфоративных язв желудка и двенадцатиперстной кишки в сравнительном аспекте с применением различных методов

\section{Материалы и метолы}

В клинике хирургии ФПК и ППС с курсом эндоскопической хирургии за период 2016-2020 гг. прооперировано 117 пациентов с ПГДЯ. Мужчин было 99 (84,6\%), женщин - 18 (15,4\%). Возраст пациентов от 18 до 65 лет, в среднем 41,1 11,4 года. Длительность симптомов до поступления в стационар составляла от 2 часов до 3 суток при средней продолжительности 2,7士1,4 дня. 101 пациент (84,2\%) были госпитализированы в течение 24 часов после появления симптомов, а остальные - до 3 дней после появления симптомов. 61 пациента (50,8\%) имели в анамнезе язвенную болезнь (ЯБ) с симптомами

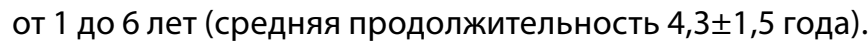
При этом 41 пациент (34,2\%) принимали нестероидные противовоспалительные препараты (НПВП) в анамнезе от 1 до 4 лет со средней продолжительностью 1,8 00,8 года. 35 пациентов (29,2\%) впервые поступили и в клинику без предшествующего приема НПВП в анамнезе.

Общими симптомами являлись сильная боль в эпигастрии, рвота и вздутие живота. 8 пациентов (6,8\%) поступили в стационар после приема наркосодержащих препаратов и употребления энергетических напитков. Помимо вышеупомянутых факторов, таких как курение, прием алкоголя, расстройство, связанное с стрес- 
Таблица 1. Характеристика обследованных пациентов

\begin{tabular}{|c|c|c|c|c|}
\hline \multicolumn{2}{|l|}{ Показатели } & $\begin{array}{l}1 \text { группа } \\
\text { (ВлО), n=56 }\end{array}$ & $\begin{array}{l}2 \text { группа } \\
\text { (ОПВ), n=61 }\end{array}$ & Р-значение \\
\hline \multicolumn{2}{|c|}{ Возраст, медиана (диапазон) [годы] } & $40,3(18-63)$ & $51,9(19-89)$ & 0,000448 \\
\hline \multicolumn{2}{|c|}{ Предоперационная задержка, медиана (диапазон) [ч] } & $4,1(0,4-13)$ & $23,7(0,5-21)$ & 0,00338 \\
\hline \multirow{5}{*}{ Класс ASA, n (\%): } & 1 & $4(7,1)$ & $1(1,5)$ & \multirow{5}{*}{0,00213} \\
\hline & $\|$ & $24(42,8)$ & $10(16,4)$ & \\
\hline & III & $25(44,6)$ & $28(45,9)$ & \\
\hline & IV & $3(5,4)$ & $17(27,9)$ & \\
\hline & $\mathrm{V}$ & 0 & $6(9,8)$ & \\
\hline \multirow{4}{*}{ Boey score, n (\%): } & 0 & $26(46,4)$ & $12(19,7)$ & \multirow{4}{*}{$<0,001$} \\
\hline & 1 & $26(46,4)$ & $20(32,8)$ & \\
\hline & 2 & $4(7,2)$ & $22(36,1)$ & \\
\hline & 3 & 0 & $7(11,5)$ & \\
\hline \multicolumn{2}{|c|}{ Продолжительность операции, медиана (диапазон) [мин] } & $97(45-149)$ & $126(54-170)$ & $<0,001$ \\
\hline
\end{tabular}

сом и инфекцией Helicobacter pylori, способствовало у 36(30,8\%) некторые другие факторы, такие как длительное голодание, что способствовало его более высокой частоте. 30 (25,6\%) пациентов имели оценку Воеу 2 или 3, что указывало на повышенный риск неблагоприятного исхода. Профиль оценки Воеу был значительно ниже у женщин по сравнению с мужчинами $(\mathrm{p}=0,036)$. При поступлении клинические признаки перитонита имелись у $80(68,4 \%)$ пациентов, без различий по полу.

Простая абдоминальная визуализация была заменена абдоминальной компьютерной томографией (КТ) в качестве метода выбора визуализации в течение периода исследования. 45 пациентов (38,5\%) прошли обычную рентгенографию брюшной полости, пневмоперитонеум был диагностирован у 98 (83,8\%) из этих пациентов. Диагноз и операция были отсрочены у 10 пациентов с исходно отрицательным рентгерологическим снимком брюшной полости.

Исследование было одобрено Комитетом по этике Дагестанского государственного медицинского университета. Были получены письменные информированные согласия пациентов на проведение исследования.

Статистический анализ проводился с использованием статистического пакета SPSS версии 15,0 для Windows (SPSS, Chicago IL, США). Для непрерывных переменных вычислялись среднее \pm стандартное отклонение (SD), медиана и диапазоны, а для обобщения категориальных переменных использовались таблицы пропорций и частот. Непрерывные переменные были классифицированы. Хи-квадрат (х2) использовался для проверки значимости связи между независимыми (предиктор) и зависимыми (результат) переменными в категориальных переменных. Для определения предикторных переменных, предсказывающих исход, был использован многомерный логистический регрессионный анализ. При вычислении р- значение считали достоверно различными при уровне значимости $(p<0,05)$.

\section{Результаты исслеАОвания}

Характеристика пациентов, подвергшихся оперативному вмешательству, представлены в табл. 1. Все пациенты для оперативного вмешательства были разделены на две группы. Первая группа 56 пациентов $(47,9 \%)$, где выполнено видеолапароскопическое оперативное вмешательство (ВЛО) и вторая группа - 61 пациент (52,1\%), которым применяли открытое оперативное вмешательство (ОПВ). Консерсия была была выявлена только у 3 (2,6\%).

Открытое оперативное вмешательство выполнялось путем верхней средней лапаротомии. Место перфорации ушивали с помощью Викрил 3-0 или капрофилом 3-0, на круглой игле по методике Оппеля-Поликарпова у 48 пациентов (41,0\%), а у 13 (21,3\%) - простым ушиванием. Размер перфорации составлял $<2,0$ см.

Лапароскопическое оперативное вмешательство проводили после ревизии брюшной полости и выявления места перфорации, последовательно ушивая швом PDS-3 или капрофилом 3-0 круглой иглой, с перевязкой сальниковой ножки к месту перфорации у 39 пациентов (69,6\%), а у 17 пациентов (30,4\%) - простым ушиванием. При всех оперативных вмешательствах лапароскопической технологии использовали экстракорпоральные методы завязывания узлов. У всех оперированных после операции проводили проверкцгерметичности швов. Операции завершали санацией брюшной полости 0,9\% физиологическим раствором и дренированием брюшной полости. 
Таблица 2. Результаты оперативного лечения пациентов в сравнительном аспекте

\begin{tabular}{|c|c|c|c|c|c|}
\hline \multicolumn{2}{|c|}{ Характеристика оперативного вмешательства } & $\begin{array}{l}1 \text { группа } \\
\text { (ВЛО), } \mathrm{n}=56\end{array}$ & $\begin{array}{l}2 \text { группа } \\
(\text { ОПВ), } \mathrm{n}=61\end{array}$ & Конвер-сия & Р-значение \\
\hline \multicolumn{2}{|c|}{ Послеоперационные осложнения, случаи } & $6(10,7 \%)$ & $27(44,3 \%)$ & 0 & 0,05 \\
\hline \multicolumn{2}{|c|}{ Без послеоперационных осложнений, случаев } & $50(89,3 \%)$ & $34(55,7 \%)$ & $3(100 \%)$ & 0,05 \\
\hline \multirow{6}{*}{$\begin{array}{l}\text { Осложнения } \\
\text { по классификации } \\
\text { Клавиена-Динго, n (\%): }\end{array}$} & Класс I & 0 & $2(3,3)$ & 0 & \multirow{6}{*}{0,004} \\
\hline & Класс II & $2(3,6)$ & $5(8,2)$ & 0 & \\
\hline & Класс IIIa & $3(5,4)$ & 0 & 0 & \\
\hline & Класс IIIb & 0 & $2(3,3)$ & 0 & \\
\hline & Класс IV & 0 & $1(1,6)$ & 0 & \\
\hline & Класс V & $1(1,8)$ & $17(27,9)$ & 0 & \\
\hline \multicolumn{2}{|c|}{ Несостоятельность шва (\%) } & $2(3,5)$ & $3(4,9)$ & 0 & 0 \\
\hline \multicolumn{2}{|c|}{ Абсцесс брюшной полости, перитонит, n (\%) } & 0 & $2(3,3)$ & 0 & 0 \\
\hline \multicolumn{2}{|c|}{ Раневая инфекция, n (\%) } & 0 & $4(6,6)$ & 0 & 0 \\
\hline \multicolumn{2}{|c|}{ Легочные осложнения, n (\%) } & $3(5,4)$ & $7(11,5)$ & 0 & 0 \\
\hline \multicolumn{2}{|c|}{ Непроходимость кишечника, n (\%) } & 0 & $1(1,6)$ & 0 & 0 \\
\hline \multicolumn{2}{|c|}{ Другие осложнения, n (\%) } & 0 & $3(4,9)$ & 0 & 0 \\
\hline \multicolumn{2}{|c|}{ Релапоротомия, n (\%) } & 0 & $2(3,3)$ & 0 & 0 \\
\hline \multicolumn{2}{|c|}{$\begin{array}{l}\text { Продолжительность пребывания в стационаре, } \\
\text { медиана (диапазон) [дни] }\end{array}$} & $5(3-14)$ & $9,8(6-31)$ & 9,3 & $<0,001$ \\
\hline \multicolumn{2}{|c|}{ Смертность, n (\%) } & $1(1,8)$ & $3(4,9)$ & 0 & 0,05 \\
\hline \multicolumn{2}{|l|}{ Всего осложнений } & $6(10,7)$ & $24(39,3)$ & 0 & $<0,001$ \\
\hline
\end{tabular}

У 85 (72,6\%) пациентов перфорация была на переднем участке первой части двенадцатиперстной кишки, а у $18(15,4 \%)$ пациентов — на желудке: 10 (8,5\%) на малой кривизне, $2(1,7 \%)$ - на большой кривизне и $2(1,7 \%)$ - на пилорической области. У 59 пациентов $(50,4 \%)$ перфорации имели минимальные размеры $(<$ 15 мм), а у 58 пациентов $(49,6 \%) \longrightarrow 19,9 \pm 0,8$ мм.

В дальнейшем все пациенты были переведены на консервативное лечение, которое включало ингибитор протонной помпы, метронидазол и амоксициллин. Наиболее распространенным послеоперационным осложнением являлся инфильтрат 7 пациентов (6,0\%). Другие послеоперационные осложнения представлены в табл. 2. Длительность пребывания в стационаре колебалась от 3 до 12 дней со средним значением

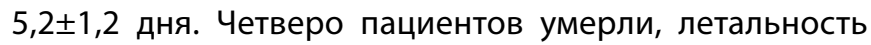
составила 6,6\%. У двух пациентов выявлено острое повреждение почек с электролитным нарушением, а еще у 2 пациентов диагностирована несостоятельность из места закрытия с развитием СВР. Результаты оперативного лечения пациентов представлены в табл. 2.

В 3-х случаях была проведена конверсия, необходимость которой была обусловлена сложностью спаечного процесса. Пациенты, которым выполнена лапароскопическая технология, были моложе, длительность симптомов была короче, с I, II и III классами по классификации ASA. Большинство пациентов с ASA III и IV класса находились в открытой группе оперативного вмешательства.
В группе видеолапароскопической технологии был один летальный исход от сердечно-сосудистой недостаточности с оценкой Воеу 3 балла. В группе ОПВ причинами смерти были сердечно-сосудистые осложнения в 2 случаях и легочные осложнения - в 1 случае. Несостоятельность шва развилась в 5 случаях (2 в группе ВЛО и 2 в группе ОПВ). Во всех случаях осложнения были выявлены с помощью дренажного контроля.

В группе ОПВ пациенту с недостаточностью шва проводилось консервативное лечение, а 2 пациентам потребовалась повторная операция. Множественный логистический регрессивный анализ с поправкой на соответствующие сопутствующие заболевания показал, что лапароскопическое оперативное вмешательство (ЛОВ) ассоциируется с меньшей продолжительностью пребывания в клинике (табл. 3).

Скорректированные нечетные коэффициенты были рассчитаны с использованием логистического регрессионного анализа. Результаты были скорректированы с учетом возраста, осложнений, класса ASA, балла MPI и времени операции.

\section{Обсужление}

Дискуссия о лапароскопической технологии по сравнению с открытым методом ПГДГ даже спустя десятилетие еще не закончена. Существуют исследования, в которых лапароскопия настоятельно рекоменду- 
Таблица 3. Логистический регрессионный анализ хирургических исходов между лапароскопической и открытой группами с соответствующими переменными

\begin{tabular}{|l|l|l|l|l|}
\hline Показатели & $\begin{array}{l}\text { Вло } \\
(\mathbf{9 5 \%} \text { ди) }\end{array}$ & $\mathbf{p}$ & $\begin{array}{l}\text { ОПВ } \\
\mathbf{( 9 5 \% ~ д И ) ~}\end{array}$ & $\mathbf{p}$ \\
\hline Классификация CD $\geq$ II & $0,33(0,07,1,50)$ & 0,150 & $0,25(0,04,1,55)$ & 0,138 \\
\hline Раняя кишечная непроходимоть & $4,08(0,55,30,32)$ & 0,170 & $4,45(0,18,111,11)$ & 0,363 \\
\hline Инфильтрат & $0,53(0,06,4,66)$ & 0,566 & $1,84(0,10,33,78)$ & 0,680 \\
\hline Пребывание в реанимации & $2,24(0,77,6,49)$ & 0,138 & $3,20(0,73,14,09)$ & 0,124 \\
\hline Пребывание в больнице $\geq 6$ дней & $0,37(0,16,0,86)$ & 0,022 & $0,15(0,04,0,54)$ & 0,004 \\
\hline
\end{tabular}

Соотношение шансов, CD: Клавьен-Диндо.

ется для лечения Пгдя [10], в то время как другие ученые делают вывод, что лапароскопическая технология имеет мало или вообще не имеет преимуществ [11].

Существуют общие опасения относительно лапароскопической технологии, которая включает работу в сложной двумерной среде с потерей восприятия глубины и тактильных ощущений наряду с ограниченным диапазоном движений. Многочисленные опубликованные исследования доказали, что приобретенные навыки помогают хирургам преодолеть эти общие проблемы лапароскопии [12].

Среди специфических проблем, связанных с лапароскопической технологией при ПгдЯ, первой выделенной проблемой была вызванная пневмоперитонеумом бактериемия [13]. Лапароскопия при перитоните может быть связана с повышенным риском развития бактериемии вследствие теоретических знаний о транслокации бактерий в системный кровоток при повышенном давлении углекислого газа в брюшной полости [13]. Однако Robinson G. отрицает эту связь, документируя отсутствие таких случаев [17]. Наше исследование также дает уверенность в отсутствии подобных случаев: бактериемия не наблюдалась ни у одного из пациентов, получавших лапароскопическое лечение.

Второй проблемой, отмеченной в первоначальных исследованиях, была большая продолжительность лапароскопической операции по сравнению с открытой методикой [14]. Более длительная продолжительность процедуры у критических пациентов означает больше шансов развития осложнений. Однако рандомизированное контролируемое исследование Siu W.et al [18]. показало, что лапароскопия на самом деле занимает меньше времени, чем открытая методика. По нашему опыту, регулярное использование лапароскопического подхода постепенно сокращало время операции и в дальнейшем приближало его к открытой методике.

Исследования Bertleff M.et.al [4], а также Luneviciusr R et.al.[13] высветили также озабоченность тем, что ча- стота несостоятельности шва в месте ушивания более распространена при лапароскопической технике сальниковой ножки, несмотря на строгие критерии повторных исследований после лапароскопии. По нашему опыту, такие случаи несостоятельности после лапароскопической технологии были только у 2 пациентов, а при открытой методике - в 3-х случаях.

С другой стороны, снижение боли и меньшая потребность в обезболивании являются наиболее согласованными факторами для лапароскопической технологии в сравнении с открытой в большинстве исследований [18]. Послеоперационную боль у пациентов с ПГДЯ, получавших лапароскопическое лечение, на основе визуальной аналоговой шкалы изучали Robencom G.et. al [17], наши исследования также показывает меньшее потребление анальгетиков и потребность в них у пациентов, получающих лапароскопическое лечение.

Tarasconi A et al. [20] сообщают о снижении послеоперационной боли, быстром выздоровлении и меньшем количестве осложнений у пациентов с ПГДЯ, получавших лапароскопическое лечение. Наше исследование также показало, что только у 10,7\%, получавших лапароскопическое лечение, имелись осложнения по сравнению с лапаротомией, где сравнительная частота была значительно выше (39,3\%), p<0,001.

Еще одними из распространенных послеоперационных осложнений являются респираторные. В нашем исследовании два пациента (3,6\%) из группы лапароскопии имели послеоперационные респираторные осложнения, что было значительно ниже по сравнению с 7 (11,5\%) пациентами из группы лапаротомии, $\mathrm{p}<0,05$.

Исследование Sauerland S. et.al.[18] показало, что к значимым факторам у пациентов, перенесших лапароскопию, следует отнести раннюю мобилизацию и возвращение к нормальной деятельности. В ходе нашего исследования мы также добились более ранней мобилизации и возвращения к нормальной активности у пациентов, получавших лапароскопическое лечение, по сравнению с лапаротомией. 


\section{ВывO $\triangle \mathrm{bl}$}

Достижения в области малоинвазивной хирургии с лапароскопической технологией дают все более многообещающие результаты, такие как меньшее вре- мя операции, меньшая потребность в послеоперационной анальгезии, более ранняя мобилизация, более короткая продолжительность пребывания в больнице и более низкая частота послеоперационных респираторных и раневых осложнений.

\section{ЛИТЕРАТУРА}

1. Керимов М.К., Коханенко Н.Ю., Луговой А.Л., Данилов С.А., Сериков В.А. Роль лапароскопии в лечении перфоративных гастродуоденальных язв. Медицина и здравоохранение. 2018, 3(4): 35-40.

2. Совцов С.А. Прогностические системы при прободных гастродуоденальных язвах. Вестник хирургии. 2016; 175(6): 114-117.

3. Bertleff M.J., Halm J.A., Bemelman W.A., et al. Randomized clinical trial of laparoscopic versus open repair of the perforated peptic ulcer: the LAMA Trial. World I Surg. 2009; 33: 1368-1373.

4. Bertleff M.J., Lange J.F. Laparoscopic correction of perforated peptic ulcer; first choice? A review of literature. Surg Endosc. 2010; 24:1231-1239.

5. Byakodi K.G. et al. Factors affecting morbidity and mortality in peptic ulcer perforation. Int Surg J. 2018; 5(4): 1335-1340. doi: 10.18203/23492902. isj20181105.

6. Seymour C.W., Liu V.X, Iwashyna T.J. Assessment of clinical criteria for sepsis», 2016;315(8): 762-774.

7. Buck D.L.M. Vester-Andersen, Moller M.H. Surgical delay is a critical determinant of survival in perforated peptic ulcer. British Journal of Surgery. 2013;100(8): 1045-1049,

8. Dodiyi-Manuel A., Wichendu P.N., Enebeli V.C. Presentation and management ot pertoraieu pepuc south Nigeria. Journal of the west African collage of surgeons. 2015; 5(3):36-48.

9. Wright G.P, A.T. Davis A.T, Koehler T, Scheeres D.E.Cost-efficiency and outcomes in the treatment of perforated peptic ulcer disease: laparoscopic versus open approach. Surgery.2014;156(4):1003-1008.

10. Gona Soro A., Kountele R. Postoperative Morbidity and Mortality of Perforated Peptic Ulcer: Retrospective Cohort Study of Risk Factors among Black Africans in Cote d'Ivoire. Gastroenterol Res Pract. 2016: 2640730. doi: 10.1155/2016/2640730.

11. Boey J., Choi, T.T. Alagaratnam, Poon A. A prospective validation of predictive factors. Annals of Surgery.1987; 205(1):22-32.

12. Kocakusak A. Does Ramadan fasting contribute to the increase of peptic ulcer perforations? Eur Rev Med Pharmacol Sci. 2017;21:150-154.

13. Lunevicius R., Morkevicius M. Comparison of laparoscopic versus open repair for perforated duodenal ulcers. Surg Endosc. 2005;19:1565-1571.

14. Wilhelmsen M., Moller M.H, Rosenstock S. Surgical complications after open and laparoscopic surgery for perforated peptic ulcer in a nationwide cohort. British Journal of I Surgery. 2015; 102(4): 382-387.

15. Moses J.F., Hughes Christopher D., Patel Pratik B. et al. Surgical Out-comes for Perforated Peptic Ulcer: A Prospective Case Series at an Academic Hospital in Monrovia, Liberia. African Journal of Emergency Medicine. 2015; 5(2):60-65. doi: 10.1016/j.aijem.2014.11.002.

16. Nirula R. Gastroduodenal perforation. Surgical Clitiics of North America. 2014; 94(1):31-34.

17. Robertson G.S., Wemyss-Holden S.A., Maddern G.J. Laparoscopic repair of perforated peptic ulcers. The role of laparoscopy in generalised peritonitis. Ann R Coll Surg Engl. 2000; 82:6-10.

18. Sauerland S., Agresta F., Bergamaschi R. et al. Laparoscopy for abdominal emergencies: evidence-based guidelines of the European Association for Endoscopic Surgery. Surg Endosc. 2006; 20:14-29.

19. Siu WT, Leong HT, Law BK, et al.: Laparoscopic repair for perforated peptic ulcer: a randomized controlled trial. Ann Surg. 2002, 235:313-319.

20. Tarasconi F., Coccolini W.L., Biffl et al. Perforated and bleeding peptic ulcer: WSES guidelines. World Journal of $\mathrm{r}^{\mathrm{Tm}}$ ttirorrv.2020; 15(1): 3.

(с) Омаров Магомед Дибирович ( maga05region1994@mail.ru ),

Магомедов Мухума Магомедович ( muxuma@mail.ru ), Магомедов Магомед Абдулхаликович ( Drmda08@mail.ru ).

Журнал «Современная наука: актуальные проблемы теории и практики» 